\section{Investigation of apomorphine during sleep in Parkinson's: Improvement in UPDRS Scores}

\author{
Miguel A. Pieroni \\ Department of Neurology, FIDES \\ Center, Jujuy, Argentina
}

\begin{abstract}
Sleep is responsible for several functions required for homeostasis. REM sleep could be a rearrangement period where limits of certain functions can be moved to a new state of balance. This study proposes that dopaminergic deficit may be responsible for the circadian dysregulation that occur with neurodegeneration and therefore a restitution of REM sleep and an improvement in Parkinson disease's symptoms can be achieved with the controlled use of dopamine agonists during the night. Twenty parkinsonian patients underwent to a onemonth study of subcutaneous nocturnal apomorphine treatment at the beginning of each REM stage. This therapeutic approach led to a significant benefit for patients in all of the 3 UPDRS scores. The mean change from baseline in the MDS-UPDRS Part I, II and III was significantly greater in the apomorphine $v s$. placebo group. In the UPDRS Part I total score was 0.8 (95\% confidence interval $[\mathrm{CI}]: 1.612,-0.012)$ and $3.3(95 \%$ CI: $4.732,1.867)$ for the placebo and apomorphine groups, respectively (difference between groups: $2.5,95 \% \mathrm{CI}: 3.454,1.545$; $\mathrm{P}=0.002$ ). For UPDRS Part II total score, the mean change was 1.3 (95\% CI: 2.692 , $0.09)$ and $4.6(6.916,2.28)$. Difference between groups: $3.3,95 \%$ CI: 4.752, 1.847 ; $\mathrm{P}=0.013$. In UPDRS Part III was $1.1(95 \%$ CI: $2.425,-0.225)$ and 5.5 (95\% CI: 8.808 , 2.191). Difference between groups: 4.4 , (95\% CI: 6.321, 2.478; $\mathrm{P}=0.012$ ). We can conclude that sleep alteration in PD can be improved by stimulation of $\mathrm{D} 2$ receptors. The symptomatic benefits obtained due to restoration of REM functions were significant.
\end{abstract}

\section{Introduction}

Neurodegenerative diseases are incurable and debilitating conditions that result in a progressive memory impairment, $\operatorname{cog}$ nitive and motor dysfunction, and behavioral disturbance. Despite its high prevalence, current treatments are unable to modify the progress of the disease. However, pathological mechanism such oxidative stress, inflammation, and neurogenesis impairment may operate differently between patients. Neurotransmitters levels are also regulated by the sleep and wake states, ${ }^{1}$ and the relationship between them and neurodegenerative disorders could be an important target to future therapeutic options. Sleep is important for homeostasis, allowing cell damages repaired, brain wastes cleared and neurotransmitters restored. It develops alternating throughout each of its cycles between slow-wave sleep (SWS) and rapid-eye-movement (REM) sleep. A mixed frequency electroencephalographic activity (theta and faster rhythms) characterized REM sleep, which arise from bidirectional interactions of cortical, hippocampal, and subcortical networks. ${ }^{2,3}$

Functionally efficient REM sleep requires a high dopaminergic activity, while its reduction or suppression can be observed when dopamine D2 receptor is blocked after a period of REM sleep deprivation. ${ }^{4}$ This selective activation of D2 receptors (but not D1) was also evaluated in dopaminergic transporter knockout (DATKO) mice allowing REM sleep recovery. 5 During waking states, with exception to GABA, brain levels of neurotransmitters are at their highest levels, while in sleep drops considerably until the end of SWS-II. During REM sleep, dopamine and glutamate reach its highest waking level. Dopaminergic neurons in the ventral tegmental midbrain are an active regulator of sleep and wakefulness states. ${ }^{6}$ Glutamatergic and cholinergic neurons of the tegmental pontomesencephalic through excitatory stimuli on the dopaminergic neurons of the ventral tegmental area reach its maximal activity during REM sleep. 7,8

Theta power in REM sleep has been correlated with post-sleep recall of recent emotional memories in humans. 9,10 Previous studies have shown the relationship between recent higher emotional intensity experiences in dreams and REM frontal theta supporting a memory consolidation processes framework. ${ }^{11,12}$ It has also been described that a predefined motor task performed during REM dreaming was associated with neural activation of the corresponding sensorimotor cortex, ${ }^{13}$ suggesting a relationship between the content of dreams and activity of certain brain regions.

\section{Objective}

During normal aging sleep is reduced and fragmented (circadian dysregulation) and this is even more pronounced in neurodegenerative disorders like Alzheimer disease (hyper-aging). ${ }^{14}$ Some studies have shown that good sleep quality could have a
Correspondence: Miguel A. Pieroni, Department of Neurology, FIDES Center, San Martin 210, San Salvador de Jujuy CP 4600, Argentina.

E-mail: miguelpieroni@hotmail.com

Key words: Apomorphine, REM sleep, Neuroplasticity, Parkinson disease, Neuromodulator effect.

Acknowledgements: The author would like to thank Natalia Colqui for their capable technical assistance.

Conflict of interest: the authors declare no potential conflict of interest.

Funding: none.

Received for publication: 14 June 2019.

Revision received: 9 September 2019.

Accepted for publication: 13 September 2019

This work is licensed under a Creative Commons Attribution NonCommercial 4.0 License (CC BY-NC 4.0).

(C) Copyright: the Author(s), 2019

Licensee PAGEPress, Italy

Neurology International 2019; 11:8207

doi:10.4081/ni.2019.8207

protective effect in those genetically susceptible individuals (APOE genotypes related) to develop Alzheimer's disease, decreasing associated neuropathological changes. ${ }^{15,16}$ Dopaminergic deficit may be partially responsible for the circadian dysregulation that occur with neurodegeneration, particularly in Parkinson's disease (PD). Studies with overnight apomorphine applied in continuous infusion were made to treat nocturnal symptoms of Parkinson. ${ }^{17}$ The continuous use of dopamine agonists throughout the night does not allow to distinguishing between effects achieved by the intervention in each stage of sleep. Moreover, some beneficial effects obtained during REM sleep could be counteracted by the effect extended to other stages and therefore not be evidenced on the results of these studies. In a previous study we showed an improvement in Parkinson disease's symptoms with a single night treatment with a dopamine agonist at the beginning of each REM. ${ }^{18}$ The current study aims to support these results extending the intervention for a period of one month. The importance of identifying and understanding the roles of REM sleep deprivation in the pathological process that underlines neurodegenerative diseases could reveal new therapeutic approaches. Apomorphine (APO) was chosen for its rapid onset of 
action (5-15 minutes) and a brief dose dependent duration of effect ( $<90$ minutes), when it is applied using the subcutaneous route of administration. It is a potent agonist of D1 and particularly of D2 receptors. Its high lipid solubility leads to transient brain concentrations, which can be up to eight times higher than plasma. ${ }^{19}$

\section{Materials and Methods}

Twenty patients with idiopathic PD from the Neurology Department of FIDES Center were enrolled in this study and divided into treatment and placebo group. ${ }^{20}$ Eligible patients were aged 40-79, with a previous diagnosis within 5 years and had at least two of the following symptoms: resting tremor, akinesia/bradykinesia and muscle rigidity (Table 1).

Data on demographic characteristics, medical history, course of disease and treatment were collected. Patients were free of severe cognitive deterioration, and had no other neurological, psychiatric or sleep pathologies (REM sleep behavior disorder or Restless Legs Syndrome). All patients were assessed under their usual medication at baseline and after 1 and 2 months of apomorphine treatment and were observed to evaluate the occurrence of any adverse drug reactions. At baseline, medication included both dopamine agonists and levodopa therapy in 19 patients, and levodopa alone in one patient. The study was approved by the ethics committee. All the patients gave their informed consent to take part in this study.

The efficacy of this approach was assessed using The Unified Parkinson's Disease Rating Scale (UPDRS). The endpoint was the change in MDS-UPDRS Part I (non-motor aspects of experiences of daily living), Part II (motor aspects of activities of daily living) and Part III (motor examination) total score from baseline to week 8 of the treatment period. Data were recorded prior to the study (BL), and follow-up visits at day $7(1 \mathrm{~W})$ and after four $(4 \mathrm{~W})$ and eight weeks (8 weeks). UPDRS I, II, III and IV Data were summarized using descriptive statistics. T test was used for paired samples and statistical analysis comparing efficacy data and quality of life between BL, 1W, $4 \mathrm{~W}$ y $8 \mathrm{~W}$.

Based on previous experience of a single night study, we proceeded to assess the results obtained in patients undergoing the same protocol during 1 month of treatment (three times a week, every week). A complete polysomnographic evaluation was made (standard electroencephalogram, electrooculography, nasal flow, respiratory efforts, electrocardiography, pulse oximetry
Table 1. Baseline patient demographics and disease characteristics.

\begin{tabular}{|c|c|}
\hline Demographics & \\
\hline $\begin{array}{l}\text { Gender } \\
\quad \text { Female } \\
\text { Male }\end{array}$ & $\begin{array}{c}6(30 \%) \\
14(70 \%)\end{array}$ \\
\hline $\begin{array}{l}\text { Age (years) } \\
<65 \text { years } \\
\geq 65 \text { years }\end{array}$ & $\begin{array}{c}64.5 \\
4(20 \%) \\
16(80.0 \%)\end{array}$ \\
\hline $\begin{array}{l}\text { Medical history } \\
\text { Time since PD diagnosis (years) } \\
\text { Dementia }\end{array}$ & $\begin{array}{c}5 \\
\text { No }\end{array}$ \\
\hline $\begin{array}{l}\text { MDS-UPDRS total score, mean (SD) at baselineab } \\
\text { UPDRS I } \\
\text { UPDRS II (activities of daily living) at "On" state } \\
\text { UPDRS III (motor examination) at "On" state }\end{array}$ & $\begin{array}{l}11,2(2,44)^{a}-11,2(2,4)^{b} \\
14,2(5,69)^{a}-14,9(6,04)^{b} \\
23,2(10,3)^{a}-24,2(10,2)^{b}\end{array}$ \\
\hline $\begin{array}{l}\text { Previous PD medication as reported at baseline } \\
\text { Levodopa } \\
\text { Dopamine agonist } \\
\text { COMT inhibitors } \\
\text { MAO-B inhibitors } \\
\text { Amantadine } \\
\text { Other oral medications }\end{array}$ & $\begin{array}{c}20(100 \%) \\
1 \\
0 \\
0 \\
0 \\
0\end{array}$ \\
\hline $\begin{array}{l}\text { Sleep-related variables; mean } \pm \text { SD } \\
\text { Sleep latency, min } \\
\text { REM latency, min } \\
\text { Sleep efficiency, } \% \\
\text { Total sleep time, min } \\
\text { Stage } 1, \% \\
\text { Stage } 2, \% \\
\text { Stage } 3, \% \\
\text { REM, \% }\end{array}$ & $\begin{array}{c}37,4 \pm 19 \\
145,5 \pm 47 \\
59,8 \pm 18,7 \\
312,7 \pm 167,5 \\
5,8 \pm 4,2 \\
64,7 \pm 8,9 \\
13,5 \pm 4,1 \\
16 \pm 5,9\end{array}$ \\
\hline
\end{tabular}

Data presented in mean \pm standard deviation (SD) or number (\%). Parkinson's disease (PD), Unified Parkinson's Disease Rating Scale (UPDRS), Catechol O-methyl transferase (COMT), Monoamine oxidase-B (MAO-B). aPlacebo; bApomorphine.

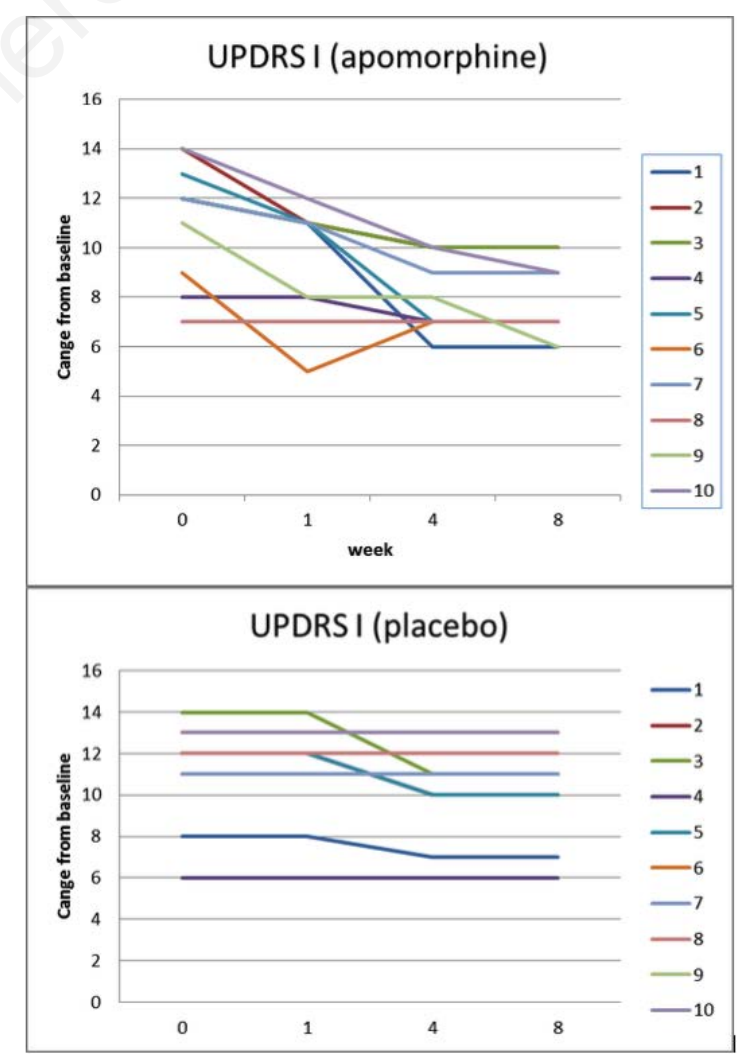

Figure 1. Total scores change from baseline over time (weeks) in UPDRS part I. Apomorphine and placebo groups. 
and electromyography) with synchronized video-EEG infrared monitoring. This study was carried out to detect timely and accurately each REM stage, the most convenient period to intervene during sleep with dopaminergic agonist effect, without extending it to other sleep stages. The stages of sleep, awakenings, alpha rhythm, respiratory events, periodic leg movements and muscle activity were scored by visual inspection in accordance with the criteria and standardized definitions.

The study involved subcutaneous administration of $2 \mathrm{mg}$ of apomorphine at the beginning of every single REM detected throughout each night recording or at regular intervals in cases where this stage did not appear. A subcutaneous access was placed for applying medication without interfering with normal sleep, using a controlled infusion pump. Only a single initial $20 \mathrm{mg}$ doses of the peripheral dopamine antagonist domperidone was given orally (to avoid potential adverse effects like QTc prolongation at higher doses) every night to control most of the side effects.

\section{Results}

The study was conducted between May and December of 2018. Of the 20 screened patients, 10 were randomized to receive placebo $(n=10)$ or apomorphine $(n=10)$. The mean age was 64.5 years. No discontinuation was registered in any of the 2 groups.

At week 8 the mean change from baseline in the MDS-UPDRS Part I total score was $0.8(95 \%$ confidence interval $[\mathrm{CI}]$ : $1.612,-0.012)$ for the placebo group and 3.3 (95\%CI: $4.732,1.867)$ for the apomorphine group, representing a relative improvement in symptoms for patients receiving apomorphine $v s$. placebo (Figure 1). The difference between groups was statistically significant (apomorphine-placebo: 2.5, 95\%CI: 3.454, 1.545; $\mathrm{P}=0.002)$. For the MDS-UPDRS Part II total score, the mean change from baseline was 1.3 for the placebo group $(95 \% \mathrm{CI}$ : $2.692,-0.09)$ and $4.6(6.916,2.28)$ for the apomorphine group (difference between groups: 3.3 , 95\% CI: 4.752, 1.847; $\mathrm{P}=0.013$ ) (Figure 2). The mean change from baseline to week 8 in the MDS-UPDRS Part III total score was $1.1(95 \% \mathrm{CI}: 2.425,-0.225)$ and 5.5 (95\%CI: $8.808,2.191)$ for the placebo and apomorphine groups, respectively (Figure 3); the between-group difference was statistically significant (difference between groups: 4.4, 95\% CI: $6.321,2.478$; $\mathrm{P}=0.012$ ). Mean changes from baseline for all individual domain scores were numerically greater for the apomorphine group than the placebo group (Figure 4).
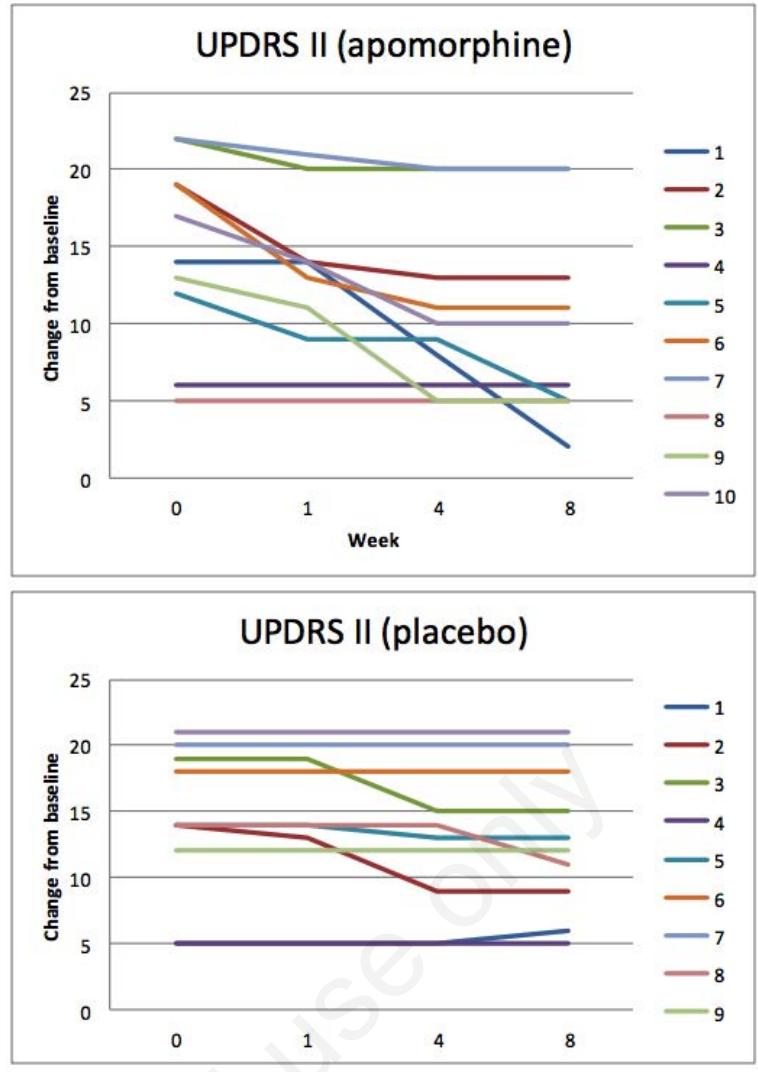

Figure 2. Total scores change from baseline over time (weeks) in UPDRS part II. Apomorphine and placebo groups.
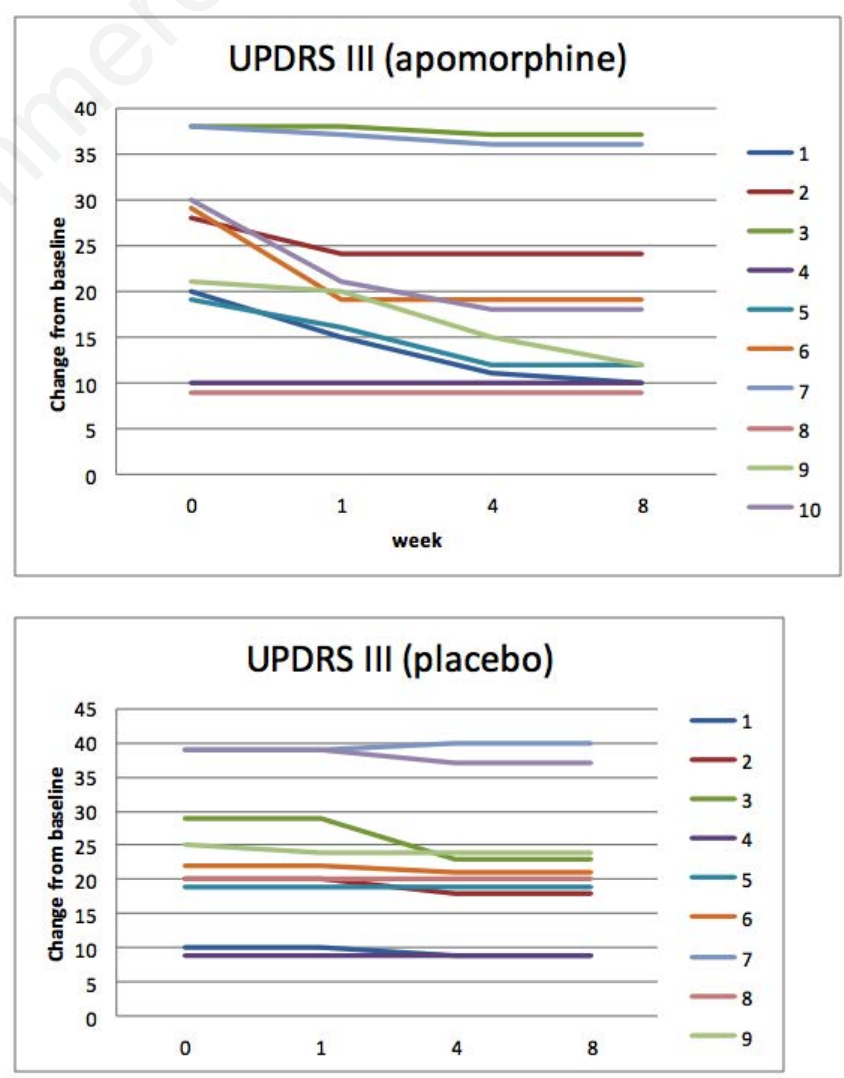

Figure 3. Total scores change from baseline over time (weeks) in UPDRS part III. Apomorphine and placebo groups. 


\section{Conclusions}

REM is a particularly complex stage that associates higher brain processing and dreams elaboration with a reduced overall activity. The typical fluctuations in physiological rhythms (heart rate, breathing, blood pressure, core body temperature and even brain electrical activity) could involve a greater plasticity state where biological limits responses are modified, allowing adaptation to the continuous changes in the environment. Muscle activity, on the other hand, can only be expressed in small muscle groups such as eye movements. From this neurophysiological viewpoint, relevant daily experiences can be recreated as conscious perceptions through neural associations reactivated by prefrontal cortex, allowing the selection of the most appropriate responses. Neuronal networks assemblies strengthened or weakened depending on the number of repetitive activations they go through. The high desynchronization of neuronal activity in REM sleep could be related to the generation and strengthening of neuronal pathways linked to recent experience. 21

This is the first time, to our knowledge, that a therapeutic approach during REM sleep is performed in patients with Parkinson's disease. Studies with overnight apomorphine applied in continuous infusion and one with patches were made to treat nocturnal symptoms of Parkinson. ${ }^{17}$ In none

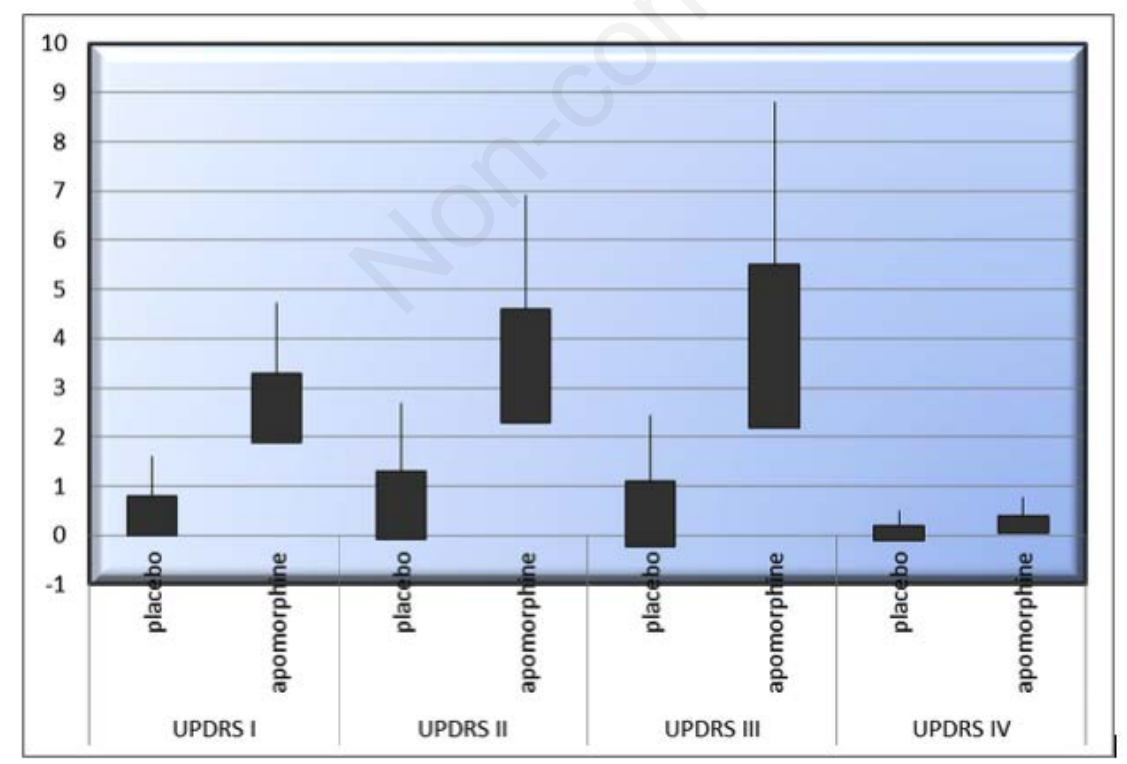

Figure 4. Comparative study between placebo and apomorphine groups. UPDRS Part I total score of $0.8(95 \% \mathrm{CI}: 1.612,-0.012)$ and $3.3(95 \% \mathrm{CI}: 4.732,1.867)$, respectively. UPDRS Part II: mean change of 1.3 (95\% CI: $2.692,-0.09)$ and $4.6(6.916,2.28)$. UPDRS Part III of 1.1 (95\% CI: 2.425, -0.225) and 5.5 (95\% CI: 8.808, 2.191). UPDRS Part VI of 0,2 (95\% CI: 0.501, -0,101) and 0,4 (95\% CI: 0.769, 0.03), respectively. of them the effects on daytime symptoms was an end point and even more, none of these was designed with the aim of assessing the benefit of dopaminergic D2 agonism in REM stage or to induce it. The results obtained are consistent with the concept that sleep disorders, particularly in REM stage, occur frequently in patients with neurodegeneration. Dopaminergic deficit causes a significant decrease of this stage, where probably is as necessary for regulation of amplitude and speed of movements as for autonomic functions. This REM reduction can be improved by stimulation of D2 receptors. Some transient postsynaptic effects and more steady plastics phenomena could explain the observed symptomatic benefit of a restored REM sleep. The first could be due to temporary up-regulation of overstimulated receptors, after dopaminergic treatment for long periods. Increased responsiveness of DA receptor has been described after short treatment of other types such as electroconvulsive therapy. ${ }^{22}$ The hypothesis of a potential neuromodulator effect of sleep on biological functions must still be confirmed by further studies. As in our previous study, REM stage developed normally once subcutaneous apomorphine was administered, or induced where REM was not observed 30 minutes after the expected time. The overall symptomatic benefits in patients with PD were significant and can only be associated to the restoration of REM functions, considering the brief dopaminergic effect limited only to this stage. This supports the hypothesis of REM sleep as a rearrangement period where limits of certain functions can be moved to a new state of balance. However, the present study has several limitations such as the relatively small number of enrolled subjects. New and more comprehensive research and longer follow-up are needed to carry out definitive conclusions and define the role of this therapy in PD and other degenerative diseases.

\section{References}

1. Everson CA, Henchen CJ, Szabo A, Hogg N. Cell injury and repair resulting from sleep loss and sleep recovery in laboratory rats. Sleep 2014;37:1929-40.

2. Saper CB, Fuller PM, Pedersen NP, et al. Sleep state switching. Neuron 2010;68:1023-42.

3. Brown RE, Basheer R, McKenna JT, et al. Control of sleep and wakefulness. Physiol Rev 2012;92:1087.

4. Datta S. Cellular and chemical neuroscience of mammalian sleep. Sleep Med 2010;11:431-40.

5. Lima MM, Andersen ML, Reksidler $\mathrm{AB}$, et al. Blockage of dopaminergic D (2) receptors produces decrease of REM but not of slow wave sleep in rats after REM sleep deprivation. Behav Brain Res 2008;188:406-11.

6. Ukponmwan OE, Rupreht J, Dzoljic M. An analgesic effect of enkephalinase inhibition is modulated by monoamine oxidase-B and REM sleep deprivations. Naunyn Schmiedeberg's Arch Pharmacol 1986;332:376-9.

7. Bartsch T, Knight YE, Goadsby PJ. Activation of 5-HT(1B/1D) receptor in the periaqueductal gray inhibits nociception. Ann Neurol 2004;56:371-81.

8. Bartsch T, Levy MJ, Knight YE, Goadsby PJ. Differential modulation of nociceptive dural input to [hypocretin] orexin a and $B$ receptor activation in the posterior hypothalamic area. Pain 2004;109:367-78.

9. Nishida M, Pearsall J, Buckner RL, Walker MP. REM sleep, prefrontal theta, and the consolidation of human emotional memory. Cerebral Cortex 2009;19:1158-66.

10. Siclari F, Baird B, Perogamvros L, et al. The neural correlates of dreaming. Nature Neurosci 2017;20:872-8.

11. Payne JD. Memory consolidation, the diurnal rhythm of cortisol, and the nature of dreams: a new hypothesis. Int Rev Neurobiol 2010;92:101-34.

12. Wamsley EJ. Dreaming and offline memory consolidation. Curr Neurol 
Neurosci Rep 2014;14:433.

13. Dresler M, Koch SP, Wehrle R, et al. Dreamed movement elicits activation in the sensorimotor cortex. Curr Biol 2011;21:1833-7.

14. Witting W, Kwa IH, Eikelenboom P, et al. Alterations in the circadian restactivity rhythm in aging and Alzheimer's disease. Biol Psychiatry 1990;27:563-72.

15. Lim AS, Yu L, Kowgier M, et al. Modification of the relationship of the apolipoprotein E4 allele to the risk of Alzheimer disease and neurofibrillary tangle density by sleep. JAMA Neurol 2013;70:1544-51.

16. Xie L, Kang H, Xu Q, et al. Sleep drives metabolite clearance from the adult brain. Science 2013;342:373-7.

17. Fernández-Pajarín G, Sesar Á, Ares B, Castro A. Evaluating the Efficacy of Nocturnal Continuous Subcutaneous Apomorphine Infusion in Sleep Disorders in Advanced Parkinson's Disease: The APO-NIGHT Study. J Parkinsons Dis 2016;6:787-92.

18. Pieroni M, Pieroni G. Novel Therapeutic Approach for Parkinson Disease during REM Sleep. J Health Edu Res Dev 2016;4:164.

19. Gronier B, Rasmussen K. Activation of midbrain presumed dopaminergic neurones by muscarinic cholinergic receptors: an in vivo electrophysiological study in the rat. $\mathrm{Br} \mathrm{J}$ Pharmacol 1998;124:455-64.
20. Hughes AJ, Daniel SE, Kilford L, Lees AJ. Accuracy of clinical diagnosis of idiopathic Parkinson's disease: a clinico-pathological study of 100 cases. J Neurol Neurosurg Psychiatry 1992;55: 181-4.

21. Voss U, Klimke A. Dreaming during REM sleep: autobiographically meaningful or a simple reflection of a Hebb'ian-based memory consolidation process? Arch Ital Biol 2018;156:99111.

22. Andersen K, Balldin J, Gottfries CG, et al. A double- blind evaluation of electroconvulsive therapy in Parkinson's disease with “on-off' phenomena. Acta Neurol Scand 1987;76:191-9. 